Original scientific paper

\title{
ANALYSIS OF LASER INTERFEROMETER MEASUREMENT UNCERTAINTY BY SIMULATING ERROR SOURCES
}

\author{
Lipus, L. C.*; Budzyn, G. ${ }^{* *} \&$ Acko, B.* \\ *University of Maribor, Faculty of Mechanical Engineering, Smetanova 17, SI-2000 Maribor, Slovenia \\ ** Faculty of Electronics, Wroclaw University of Science and Technology, \\ Janiszewskiego 7, 30-370 Wroclaw, Poland \\ E-Mail: lucija.lipus@um.si,grzegorz.budzyn@pwr.edu.pl,bojan.acko@um.si
}

\begin{abstract}
The article presents measurement uncertainty analysis of accurate one-dimensional measurements with laser interferometers in precise industrial production and in calibration laboratories. To enable high measurement accuracy, environmental conditions shall be well controlled and laser interferometers, including air sensors, shall be calibrated periodically. Additionally, for calibration of measurement instruments and coordinate measuring machines, a positioning system, such as a video probe, shall be evaluated. In the presented research, the influencing parameters were observed on specific cases in laboratory measurement conditions, as examples of calibration systems that are applied in the accredited metrology laboratory (Laboratory for Production Measurement, Faculty of Mechanical Engineering). Through the review of possible error sources, which can be categorized into three groups - instrumental, environmental, and geometrical errors of the measuring system, the uncertainty budget was summarized with the aim to recognize and reduce the most influencing components and reach a proper measurement accuracy in real production environment.

(Received in February 2021, accepted in April 2021. This paper was with the authors 3 weeks for 1 revision.)
\end{abstract}

Key Words: Laser Interferometry, Measurement Uncertainty, Simulation, Calibration

\section{INTRODUCTION}

Displacement lasers are widely employed for precise dimensional measurements due to their high resolution and outstanding accuracy. Measurement accuracy of these systems generally exceeds the requirements of the manufacturing tolerances, while used for monitoring advanced precise manufacturing technologies such as automotive industry, either for direct workpiece geometry measurements or for calibrating very accurate measuring instruments. In all cases, an accuracy analysis of the total measurement performance is needed [1]. Furthermore, the measurement systems shall be ready for fully automated integration into advanced manufacturing systems by enabling direct transformation of measurement data including calibration results into global information systems [2].

Laser interferometry is based on the principle of Michelson interferometer (Fig. 1 a): a beam splitter divides a highly stabilized light beam into two beams, the reference beam is returned by a fixed reflector and an another one returns from the mirror which is shifted along the measured path. Combined by the splitter, they interfere into pulses, which are counted by a photo-electro detector. To determine if the movement was in positive or negative direction, two beams are used in real construction: in a homodyne system, the beam from one-mode laser (with one frequency of light) is divided into two beams by the splitter, polarizing them vertically and horizontally; while in a heterodyne system, two-mode laser (with frequency of light $v_{1}$ polarized vertically and $v_{2}$ polarized horizontally) is used (Fig. $1 \mathrm{~b}$ ). The detector counts the number of wavelengths and partial wavelengths while the mirror is being displaced (equivalent to the Doppler effect frequency $v_{\mathrm{D}}$ ). The vacuum wavelength is dynamically corrected for the ambient conditions along the optical path, measured with a precise air sensor.

Through the review of possible error sources in laser interferometric systems, they can be classified into three categories: geometrical errors, environmental errors, and instrumental 
errors [3]. To recognize and reduce the most influencing components and reach proper measurement accuracy in real environment, all possible sources of errors will be simulated and summarized into uncertainty budget for a practical case from our laboratory. In order to be able to reach expected measurement accuracy, laser interferometers and corresponding air sensors shall be calibrated periodically [4]. The laser frequency is calibrated by comparison with primary standard laser [5-7], while whole instrumental system can be checked by means of comparison measurement with a reference laser interferometer, presented in this article.

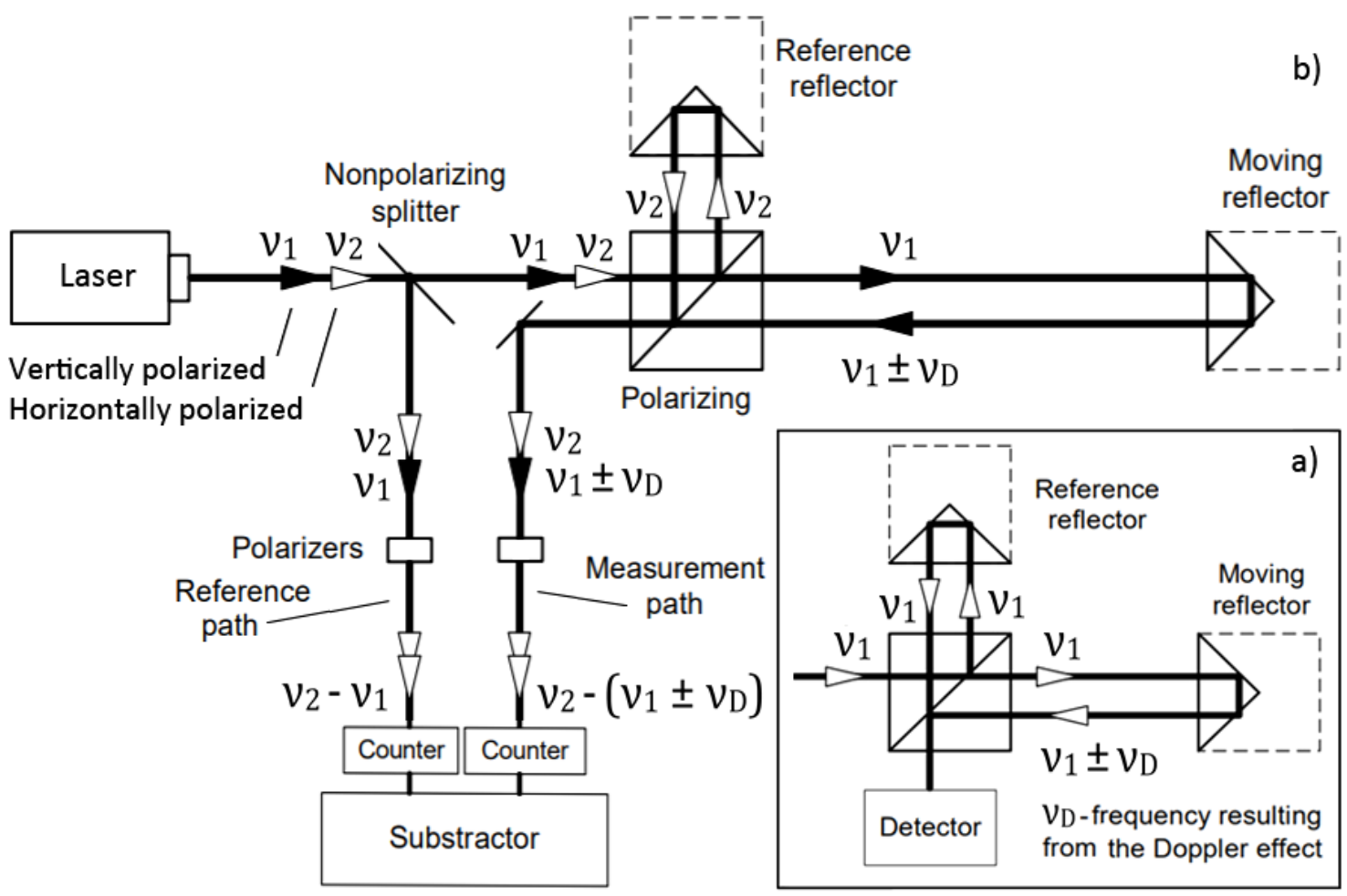

Figure 1: A configuration of interferometer system: a) Michelson's interferometer, b) Heterodyne interferometer [3].

\section{EVALUATION OF CONTRIBUTIONS TO THE MEASUREMENT UNCERTAINTY}

There are four significant groups of contributions to the total measurement uncertainty of an interferometric measurement: geometrical errors, environmental errors, material temperature errors, and instrumental errors. They were evaluated by means of analytical calculations, experiments, and simulation of real measurement conditions. Simulations were used for estimating intervals of residual errors after correcting the measurement result for systematic errors.

\subsection{Geometrical errors}

Cosine error occurs when the laser beam path and the axis of the stage motion are not completely parallel. This results in an error between the measured distance, i.e., laser interferometer's reading ( $L_{\mathrm{LI}}$ - distance along the beam axis) and the actual travelled distance ( $L$-displacement along the stage axis). Due to angle $\varphi$ between these axes (Fig. 2 a), the error is negative and proportional with the measured displacement:

$$
e_{\cos }=L(\cos \varphi-1) \approx-L \frac{\varphi^{2}}{2}
$$


Abbe error $\left(e_{\text {abbe }}\right)$ occurs due to sloping of the reflector during measurements and depends on probe's clamping and straightness of the stage axis. It is related to the distance between the beam path axis and the axis of stage motion ( $h_{A b b e}-$ Abbe offset, Fig. 2 b):

$$
e_{\text {Abbe }}=h_{\text {Abbe }} \tan \varphi
$$

$L_{\mathrm{LI}}=$ distance along the beam axis

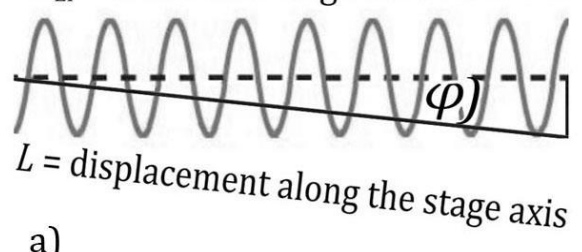

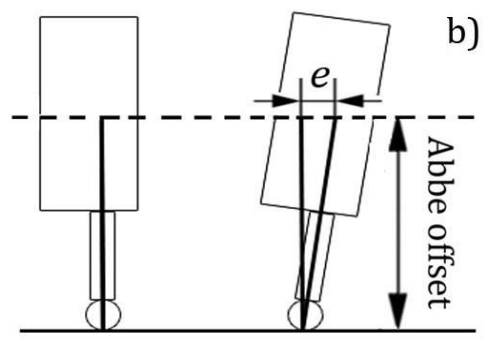

Figure 2: Geometrical errors: a) Cosine error, b) Abbe error.

Abbe error caused by the pitch of the moving part of the device carrying the probe (Fig. 2 b) can be corrected by applying angle measurements performed with laser interferometry or with an electronic level in fix points, e.g., in the zero position and in the end point of the measurement path. With highly precise positioning of a video probe system along a long measurement path, $10 \mathrm{~m}$ for instance, this type of error can contribute up to few $\mu \mathrm{m}$; while in short precise measurements, even random errors of few-ten $\mathrm{nm}$ caused by uncontrolled mechanical changes along the axis, can contribute essentially to the total measurement uncertainty $[1,8]$.

To minimize the geometrical errors, it is important to carefully align the laser beam over the entire path of the machine stage, by proper alignment procedures for each type of interferometric system. In the case of single pass configuration (Fig. 3), firstly the laser head is aligned by parallel shifts for targeting the beam into the target centre on the mirror in a close position, and by angle adjustments in the mirror's maximum displacement.

a)

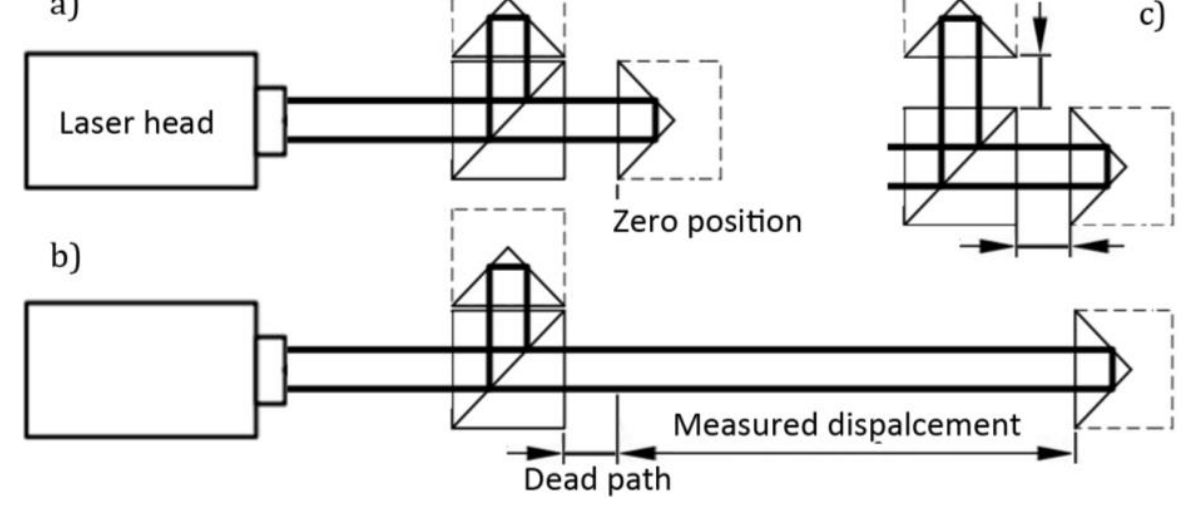

Figure 3: A configuration for minimizing the death path: a) and b) zero position close to the splitter, c) similar distance of reflectors to the splitter.

Secondly, to assure that the beam returns along the same path (or a parallel path when using a reflector prism) from the mirror into the detector, the angle of the mirror is adjusted at the maximum mirror displacement, in order to target the returning beam into the entrance hole of the laser head. Finally, the interferometer prism is installed on the optical path as close as possible to the starting point of displacement measurement path (zero-point position, Fig. 3 a) and adjusted to reach maximum power of the returned beam.

For minimising the cosine error $\left(u_{c o s}\right)$, we choose the maximum distance for adjusting the laser beam parallel to the displacement path axis. At a measurement length of $10 \mathrm{~m}$, visual 
adjustment of the beam centre within an error band of $\pm 1 \mathrm{~mm}$ would result in $\varphi=10^{-4}$ (Fig. $2 \mathrm{a}$ ) and consequently the uncertainty along the path would be $u_{\cos }=0.005 \cdot 10^{-6} \cdot \mathrm{L}$, as calculated by Eq. (1), which is negligible. For shorter measurement path of e. g. $2 \mathrm{~m}$, a precise beam adjustment within an error band of $\pm 0.5 \mathrm{~mm}$ at this maximal distance would result in $\varphi=2.5 \cdot 10^{-4}$ and $u_{\cos }=0.031 \cdot 10^{-6} \cdot L$. This strong dependence of cosine error on the adjusting distance is presented in Fig. 4.
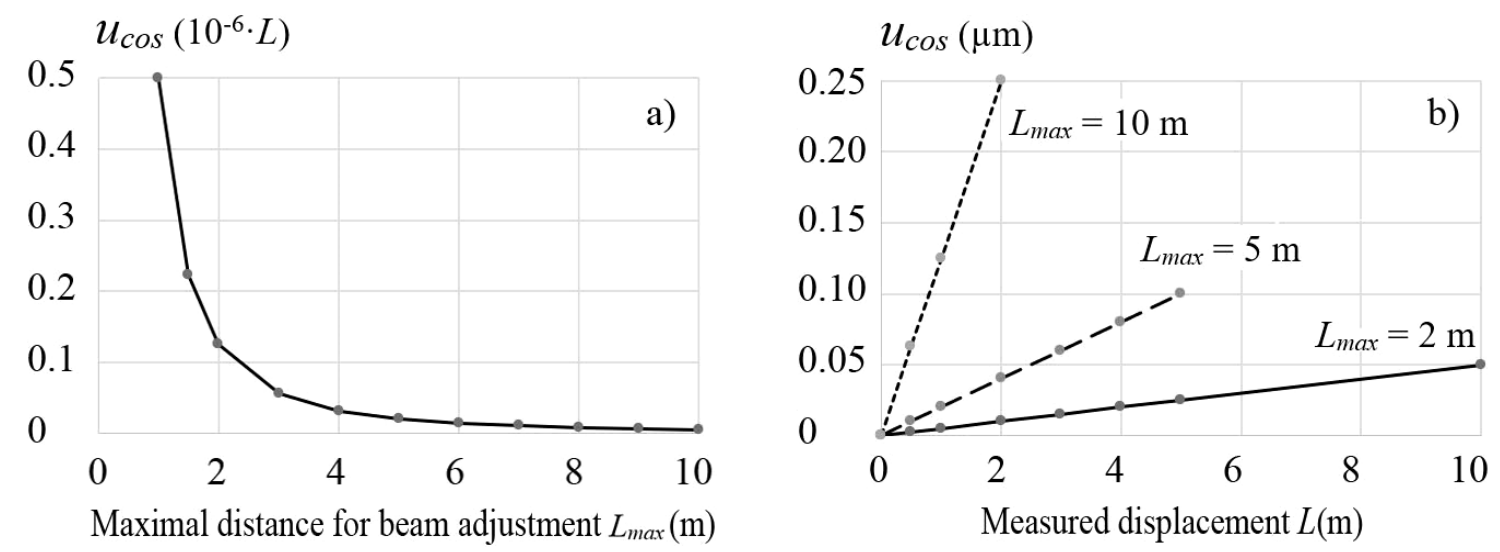

Figure 4: A simulation of a cosine error (at adjusting the beam centre within error band of $\pm 1 \mathrm{~mm}$ ): a) depending on maximal possible distance for minimizing angle error, b) depending on measured displacement at different adjusting distances.

\subsection{Environmental errors}

Measurement uncertainty due to environmental effects is related to the dependence of the light wavelength on the atmospheric conditions and on the thermal properties of the machine under test. The temperature change of the optics during the measurement can also contribute to the measurement uncertainty in minor extend.

The wavelength, generally given in the laser's specifications and calibration certificates, is the value in vacuum: $\lambda_{\mathrm{v}}=c / v$; where $c$ is the light speed in vacuum. Since the laser interferometric system normally operates in atmospheric conditions, the counted number of wavelengths along the displacement depends on $\lambda$ value in the air and must be compensated with $n$, the air refractive index: $\lambda=\lambda_{\mathrm{v}} / n$.

The air index depends mostly on the pressure $p$, temperature $T$ and humidity $H$, and was empirically determined by Edlen equation, updated by Birch and Downs [9]. Software is available online by NIST (National Institute of Standards and Technology) [10] with updated Edlen equation and Ciddor equation, which includes the correction for carbon dioxide content [11]. Close to the reference air conditions $\left(p_{0}=1013.25 \mathrm{hPa}, T_{0}=20^{\circ} \mathrm{C} ; H_{0}=50 \%\right.$; and carbon dioxide content $\left.x_{0}=450 \mathrm{ppm}, n_{0}=1.000271373 \pm 23 \cdot 10^{-9}\right)$, the sensitivity coefficients are: for air temperature $\delta n / \delta T=-0.955 \cdot 10^{-6} /{ }^{\circ} \mathrm{C}$; for air pressure $\delta n / \delta p=0.268 \cdot 10^{-6} / \mathrm{hPa}$; for humidity $\delta n / \delta H=-0.0085 \cdot 10^{-6} / \%$; and for carbon dioxide content $\delta n / \delta x=1.5 \cdot 10^{-10} / \mathrm{ppm}$. A laser interferometer system uses software for correcting the wavelength in environmental conditions. There can be quite some difference between manufacturers and software versions, thus, the software shall be checked whether the refractive index equation is used properly by the system software [4].

Without wavelength compensation for real air condition, but just assuming a standard and homogeneous air composition, an essential error occurs even in well stabilized air conditions. The number of counted wavelengths is related to the wavelength value in real air condition, while the displayed result is calculated by using an uncompensated wavelength value. For example, at temperature variation of $\pm 1{ }^{\circ} \mathrm{C}$ around the standard temperature, the error is $\Delta L / L=\Delta n / n_{0} \approx \Delta T(\delta n / \delta T)=1 \mu \mathrm{m} / \mathrm{m}$ of a measured displacement. 
After wavelength compensation, some measurement uncertainty still remains due to the sensitivity coefficients of an air sensor and nonhomogeneous air conditions along the optical path. Air sensor shall be calibrated periodically in order to check possible drift in deviation and sensitivity. The deviation is recommended to be used as a correction value in the software for wavelength compensation, while the instrumental uncertainty shall comprehend both the maximum drift during calibration periods and the sensitivity (measurement uncertainty of the calibration).

In our practical application, a calibration certificate for the thermometer gives us the deviation (at standard conditions) with expanded uncertainty of $U=(-0.1 \pm 0.2)^{\circ} \mathrm{C}$; and from successive certificates the maximum drift of $\pm 0.1^{\circ} \mathrm{C}$. Similarly, from calibration certificate of the barometer, we get $(0.4 \pm 0.2) \mathrm{hPa}$ and the maximum drift $0.4 \mathrm{hPa}$. For humidity, we simply assume variation along the optical path less than $\pm 20 \%$.

The uncertainties are then computed in accordance with the Guide to the expression of uncertainty in measurement [12]. The uncertainty budget consists of uncertainty components evaluated as Type B. A maximum possible error reduced by $\sqrt{2}$ at assumed normal probability distribution, or by $\sqrt{3}$ at assumed rectangular distribution.

The combined uncertainty is calculated as a root of sum squares of the uncertainty components. Finally, the uncertainty is then multiplied by a coverage factor $2(U=2 u)$, providing a level of confidence of approximately $95 \%$.

Assuming a rectangular distribution, drifts are divided by $\sqrt{3}$ and standard uncertainty is calculated as a half expanded uncertainty. The index uncertainties of each air parameter are for this case calculated by Eqs. (3) to (5) and the uncertainty of the wavelength compensation is calculated by Eq. (6):

$$
\begin{gathered}
u_{T}=\sqrt{\left(\frac{0,1}{\sqrt{3}}\right)^{2}+\left(\frac{0,2}{2}\right)^{2}} \cdot 0.955 \cdot 10^{-6} \cdot L=0.11 \cdot 10^{-6} \cdot L \\
u_{p}=\sqrt{\left(\frac{0,4}{\sqrt{3}}\right)^{2}+\left(\frac{0,2}{2}\right)^{2} \cdot 0.268 \cdot 10^{-6} \cdot L=0.07 \cdot 10^{-6} \cdot L} \\
u_{H}=\frac{20}{\sqrt{3}} \cdot 0.0085 \cdot 10^{-6} \cdot L=0.10 \cdot 10^{-6} \cdot L \\
u_{\text {sensor }}=\sqrt{u_{T}^{2}+u_{p}^{2}+u_{H}^{2}}=0.16 \cdot 10^{-6} \cdot L
\end{gathered}
$$

where:

$$
\begin{array}{ll}
u_{T} & - \text { uncertainty due to temperature evaluation, } \\
u_{p} & - \text { uncertainty due to pressure evaluation, } \\
u_{h} & - \text { uncertainty due to humidity evaluation, } \\
u_{\text {sensor }} & \text { - uncertainty due to total evaluation of environmental parameters, } \\
L & - \text { measured length. }
\end{array}
$$

In the case of essential temperature and pressure variation along the optical path, while using just one air sensor, additional index uncertainties shall be included in Eqs. (3) and (4). At variations in well controlled air conditions $\left( \pm 0.5^{\circ} \mathrm{C}, \pm 0.5 \mathrm{hPa}\right.$, and $\pm 10 \% \mathrm{Rh}$ for instance) we get $u_{\text {var } T}=0.28 \cdot 10^{-6} \cdot L ; u_{v a r p}=0.08 \cdot 10^{-6} \cdot L$; while the value in Eq. (5) remains the same, thus the uncertainty of the wavelength compensation, Eq. (6), is around double now: $u_{\text {air }}=0.32 \cdot 10^{-6} \cdot \mathrm{L}$.

For simulating air temperature $\pm \Delta T$ (Fig. 6), at semi-controlled air pressure $\pm 1 \mathrm{hPa}$, and humidity variation of $\pm 20 \%$, the following general expression can be derived:

$$
u_{L I}=\sqrt{0.23^{2}+\left(0.55 \mathrm{~K}^{-1} \cdot \Delta T\right)^{2}} \cdot 10^{-6} \cdot L
$$


where:

$u_{L I}$ - uncertainty of the laser interferometer indication due to air parameters' evaluation,

$L \quad$ - measured length.

Dead path error is caused by an uncompensated length of the laser beam between the interferometer and the retroreflector, with the machine stage at zero position (Fig. $3 \mathrm{~b}$ ). It can occur when environmental conditions vary during the measurement but can be eliminated by placing the linear interferometer optics as close to the zero point of the moveable reflector as possible.

For semi-controlled air (with time variations $\pm 0.5^{\circ} \mathrm{C}$ and $\pm 1 \mathrm{hPa}$ and $\pm 20 \%$, $u_{\text {air }}=0.36 \cdot 10^{-6} \cdot \mathrm{L}$ ), the error is negligible if the dead path is less than $10 \mathrm{~mm}$. When the zero position cannot be set so close to the splitter, a possible solution is to install the fixed reflector at similar distance to the splitter as the moving reflector at zero point (Fig. $3 \mathrm{c}$ ) and to provide homogenous air conditions in that area.

A usually underestimated component of the environmental errors is the influence of the air propagation through the turbulent atmosphere. This phenomenon has been extensively evaluated and described in $[13,14]$, yet mostly for very large distances and applications in astronomy or communication. Recently published tests [15] performed on shorter distances are showing great dependence of the laser beam position on the air density fluctuations over the laser paths.

In similar laboratory conditions, the measurements were repeated, and the distance fluctuations caused by air changes were tested. The measurement setup was the same as the one in Fig. $3 \mathrm{~b}$ with the distance between the reference element and the measurement elements being changed from $0.05 \mathrm{~m}$ to $4.6 \mathrm{~m}$. The results presented in Fig. 5 proof that the longer the laser path is, the less accurate is a distance measurement in industrial conditions.

For standard industrial applications, the observed slope of fluctuations $0.04 \cdot 10^{-6}$ do not pose a problem, while in laboratory measurements, the air conditions along the optical path need to be well controlled in order to ensure that fluctuations are negligible, as presented for our laboratory case.

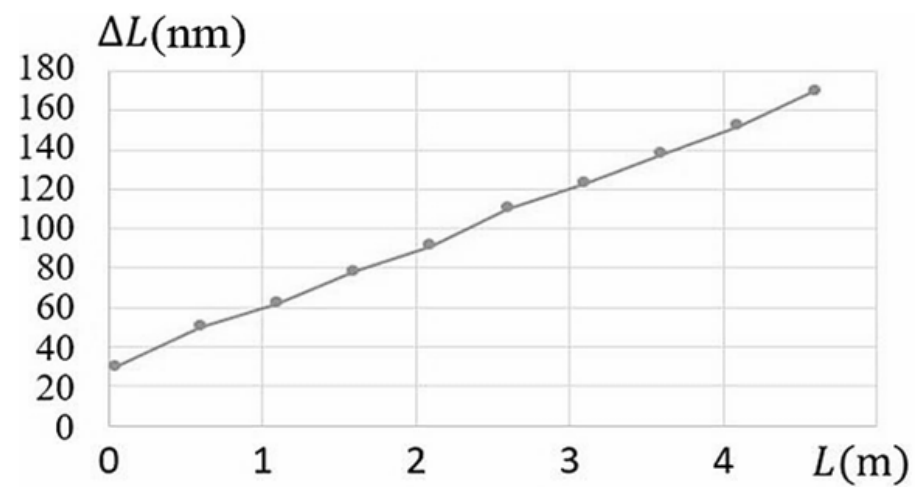

Figure 5: Fluctuations $\Delta L$ of measured distance $L$ caused by the air changes.

\subsection{Material temperature errors}

In real production conditions, extremely high material temperature gradients may occur [16] but even at less invasive treatments generally stabilisation is crucial for reaching temperature conditions that are acceptable for precise measurements. Such reduced material temperature deviations can still have significant contribution to the measurement uncertainty.

The uncertainty of the measured length $L$ due to a material temperature deviation is estimated in Eq. (8), where $\theta$ is the deviation from the reference temperature $20^{\circ} \mathrm{C}$ and $\alpha$ is the temperature expansion coefficient of the material. Depending on both parameters, $\alpha$ and $\theta$, the 
measurement uncertainty $u_{\text {mat }}$ is expressed by Eq. (9) with partial uncertainties and partial derivatives, according to the definition in Eq. (11).

Taking an example of $\alpha=(10 \pm 2) \cdot 10^{-6} \mathrm{~K}^{-1}$ for steel, we get $u_{\alpha}=2 \cdot 10^{-6} / \sqrt{3} \mathrm{~K}^{-1}$, $c_{\alpha}=\delta e / \delta \alpha=L \cdot \theta$, and $u_{\theta}=\theta / \sqrt{3}, c_{\alpha}=\delta e / \delta \theta=L \alpha=10 \cdot 10^{-6} \cdot L$.

$$
\begin{gathered}
e_{\text {mat }}=L \cdot(1+\alpha \theta) \\
u_{\text {mat }}=\sqrt{\left(u_{\alpha} \cdot c_{\alpha}\right)^{2}+\left(u_{\theta} \cdot c_{\theta}\right)^{2}}=5.9 \cdot 10^{-6} \mathrm{~K}^{-1} \cdot \theta \cdot L
\end{gathered}
$$

where:

$u_{\text {mat }}$ - uncertainty due to material temperature,

$u_{\alpha} \quad$ - uncertainty of the thermal expansion coefficient,

$u_{\theta}$ - uncertainty of the material temperature evaluation,

$c_{\alpha}-$ sensitivity coefficient of the thermal expansion coefficient,

$c \theta-$ sensitivity coefficient of the material temperature,

$L \quad$ - measured length.

In sub-micrometre accurate measurements, where temperature needs to be stabilized within $\pm 0.1 \mathrm{~K}$, the interferometry and the material temperature components get comparably important [17]. Simulation of these temperature-dependent uncertainty components at higher temperature deviations, we find that the interferometry component, Eq. (7), is negligible in comparison with the component due to the material temperature deviation, Eq. (9), as presented in Fig. 6.

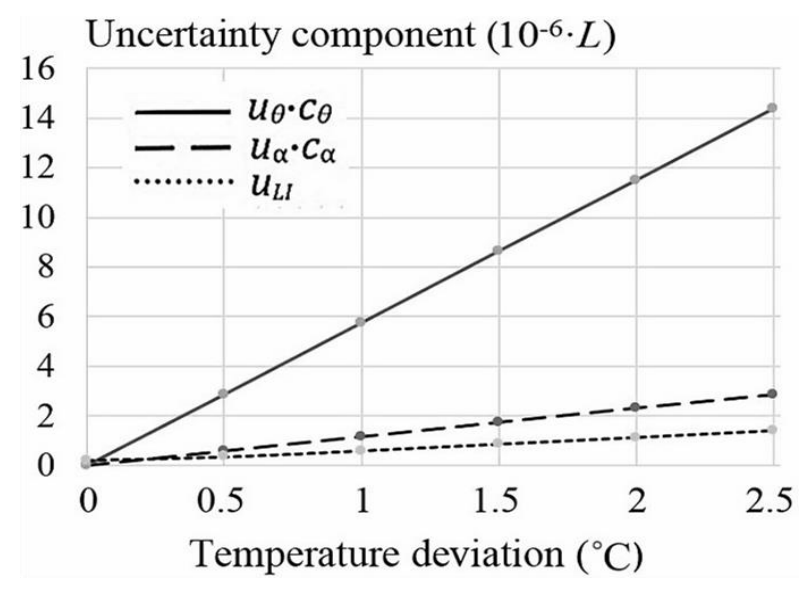

Figure 6: A comparison of temperature-dependent uncertainty components.

\subsection{Instrumental errors}

The instrumental error sources are intrinsic to the laser system: electronic-counting system, sampling time, software algorithms (for wavelength compensation by Edlen equation for instance) and for material temperature expansion.

The frequency shall be periodically calibrated, in order to check the frequency stability and to yield the correction for the nominal vacuum wavelength value in the software [6]. In stabilized HeNe two-mode lasers, used in heterodyne systems, the frequencies of vertically and horizontally polarized component usually differ in a range of (1.5 to 2) $\mathrm{MHz}$, which would contribute in up to $0.004 \cdot 10^{-6} \cdot L$ difference in measured results.

After a proper warming time, the stability of such lasers is such that in comparison to other errors its contribution is negligible, less than $0.001 \cdot 10^{-6} \cdot L$ (calculated from $U=0.5 \mathrm{MHz}$ ). With years, drift of frequency from the previous nominal value occurs, which can essentially contribute to the error in wavelength correction; for instance, a small drift of $2 \mathrm{MHz}$ contributes an error $0.004 \cdot 10^{-6} \cdot L$. 
Total instrumental error can be evaluated by direct comparison of tested laser with the reference laser by aligning the laser along the same optical path (Fig. 7). Other influencing errors (geometrical and environmental errors) are eliminated by the following procedure.

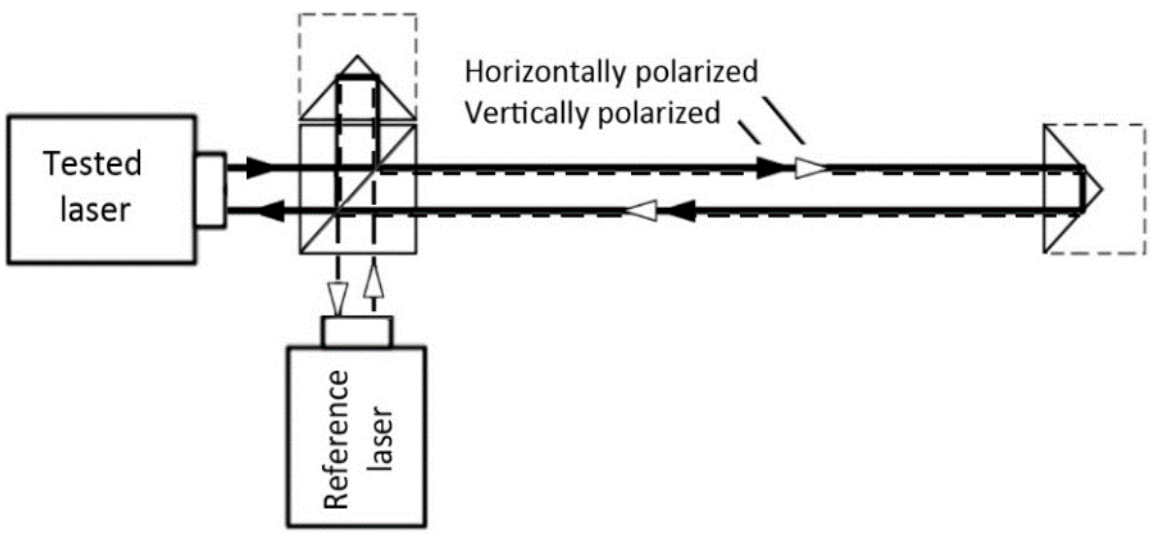

Figure 7: A configuration for two lasers comparison.

The tested laser shall be aligned along the measurement axis and optics shall be adjusted to minimize the cosine error. Then the reference laser is aligned at fixed optics. Vertically polarized component of the tested laser passes the splitter and is returned on the optical path into the detector, while the horizontally polarized component is directed to the reference reflector and is reflected in the splitter back into the detector. Oppositely, the horizontally polarized component from the perpendicularly placed reference laser is directed onto the optical path, while the vertically polarized beam travels back as a reference component. In such way, lasers beam travels along the same measurement path but does not interfere due to different polarizations. In such composition we observe the vertical component of the tested laser, while knowing both components of the reference laser.

In our experiment of direct comparison measurements, the laser interferometers were aligned precisely following the described procedure, in order to eliminate geometrical errors, while the software parameters were adjusted to standard values in order to eliminate environmental errors.

By carefully aligning the beam with a maximum deviation from the reflector centre of $1 \mathrm{~mm}$ per $10 \mathrm{~m}$ distance, the remaining cosine error is less than $0.005 \cdot 10^{-6} \cdot \mathrm{L}$ for each laser, Eq. (1), thus multiplied with $\sqrt{2}$ for both together is $0.007 \cdot 10^{-6} \cdot \mathrm{L}$.

Abbe error, Eq. (2), is lower than $1 \mu \mathrm{m}$ due to the offset of the beams along the measurement axis, but is equal for both lasers, because they travel through the same reflector. It is therefore eliminated from the comparison of measured displacements.

Similarly, the dead path is the same for both lasers and thus theoretically eliminated, but still the zero point shall be started as close as possible to the splitter and simultaneously activated in the software of both lasers in order to eliminate the difference of few $\mathrm{nm}$, as it was estimated for semi-controlled air conditions in section 2.2. In order to minimize the difference, we can use similar counting period by choosing the smallest period in the software for both lasers.

To eliminate environmental influences, the comparison is done without the air sensor, simply adjusting the air parameters and the material temperature to reference values in the software. In such way, both lasers give equally "wrong" results that are nullified in the comparison result.

The zero point was activated simultaneously at a short death path. For the reference laser, the nominal wavelength in the software was rewritten with the value from the calibration certificate. To avoid variating numbers on the $\mathrm{nm}$ decimal place, the resolution of display was 
reduced to $10 \mathrm{~nm}$, which contributes to standard uncertainty of $10 \mathrm{~nm} / \sqrt{3}=6 \mathrm{~nm}$, which is noticeable only at small displacements.

At each $1 \mathrm{~m}$ displacement along a $10 \mathrm{~m}$ optical path, pairs of 10 readouts ( $L_{\text {tested }}$ and $L_{\mathrm{ref}}$ ) were collected, and the deviation $\Delta L$ was calculated, showing a linear correlation with the displacement $L$ (Fig. 8):

$$
\Delta L=L_{\text {tested }}-L_{\text {ref }}=\Delta L_{L=0}+k \cdot 10^{-6} \cdot L
$$

where:

$\Delta L \quad$ - deviation in length,

$L_{\text {tested }}$ - length, indicated by the tested laser interferometer,

Lref - length, indicated by the reference laser interferometer,

$k \quad-$ instrumental uncertainty coefficient,

$L \quad$ - measured length.

$\Delta L(\mu \mathrm{m})$

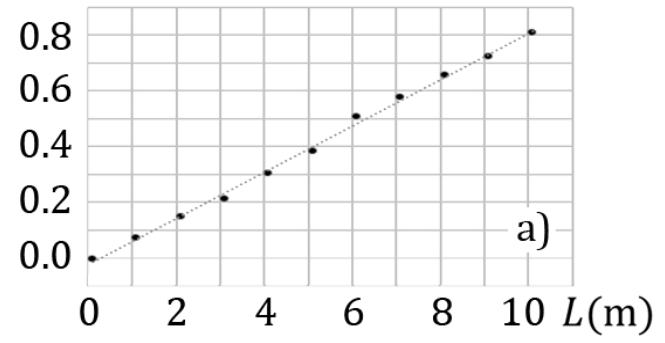

$\Delta L(\mu \mathrm{m})$

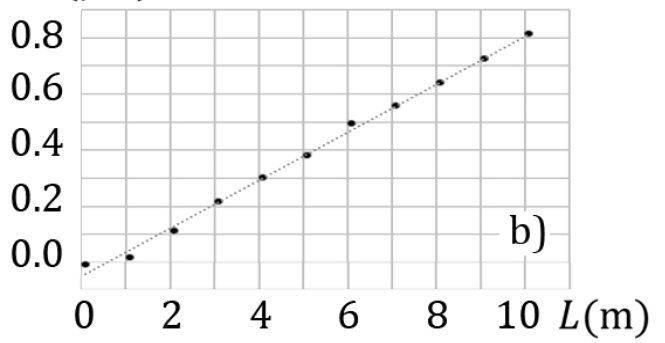

Figure 8: Evaluation of coefficient $k$ from deviation of measured displacements; a) at shifting from zero to maximal length position (slope $k=0.083$ ), b) at returning direction (slope $k=0.085) ;(100 \%$ beams' return, display with nm resolution).

The pairs inside the sequences of 10 readouts (at each $1 \mathrm{~m}$ displacement) has shown a variation of $0.045 \cdot 10^{-6} \cdot \mathrm{L}$, while the average values for sequences along a $10 \mathrm{~m}$ path have demonstrated strong linear correlation with a similar slope in both displacement directions. Considering the higher slope value, the instrumental uncertainty component of the tested laser is lower than $0.085 \cdot 10^{-6} \cdot \mathrm{L}$.

\section{DISCUSSION OF RESULTS}

The most important environmental error source is the temperature change along the base of the measured machine, causing variation of the machine dimensions. They can be significantly reduced by stabilizing the temperature and by using materials with low temperature expansion. These errors can also be numerically compensated by using proper integrated software. Depending on the configuration and expandability of the machine components, more than one temperature sensor may be used for the corrections of measurement results, in order to achieve better accuracy.

Possible sources of errors for an example of precise measurement with a laser displacement interferometer in well controlled air conditions are summarized in Table I.

The measurement error is evaluated by Eq. (11), where the length $L$ is the measured probe displacement, while $\theta$ is the material temperature deviation from $20^{\circ} \mathrm{C}$. The length measured by laser interferometer $L_{L I}$ is corrected by using Edlen equation.

Total measurement uncertainty $u_{\text {tot }}$ is determined by Eq. (12), with partial uncertainties $u_{i}$ and their sensitivity coefficients $c_{i}=\delta e / \delta x_{i}[18,19]$.

After proper alignment of the whole mechanical and interferometer displacement system, as described before, the geometrical errors of the interferometer system (the cosine, Abbe and the death path errors) are negligible (as presented in section 2.1, and for dead path error in 
section 2.2), while the probe positioning error (the camera's resolution and the probe's Abbe error) contributes on the level of a few tens of nanometres [1].

The uncertainty equation for laser interferometry displacement includes the sensor's sensitivity, well controlled air conditions regarding pressure and air humidity, while the temperature deviation was taken as a simulation parameter (as described in section 2.2), and the uncertainty of the instrumental counting system also contributes some error in the zeropoint (as observed in section 2.4).

$$
\begin{gathered}
e=L \cdot(1+\alpha \theta)-L_{L I}+e_{c o s}+e_{\text {DeadPath }}+e_{\text {Abbe }} \\
u_{\text {tot }}^{2}=\sum_{i}\left(u_{i} \cdot c_{i}\right)^{2}
\end{gathered}
$$

\begin{tabular}{|c|c|c|c|}
\hline$x_{\mathbf{i}}$ & $\boldsymbol{u}_{i}$ & $c_{i}$ & $u_{i} \cdot c_{i}$ \\
\hline$L$ & $25 \mathrm{~nm}$ (resolution) & $(1+\alpha \theta) \approx 1$ & $25 \mathrm{~nm}$ \\
\hline$e_{\text {Abbe }}$ & $\leq 35 \mathrm{~nm}$ & 1 & $35 \mathrm{~nm}$ \\
\hline$\alpha$ & $2 \cdot 10^{-6} / \sqrt{3} \mathrm{~K}^{-1}$ & $L \theta$ & $1.15 \cdot 10^{-6} \mathrm{~K}^{-1} \cdot \theta \cdot L$ \\
\hline$\theta$ & $\theta / \sqrt{3}$ & $L \alpha=L \cdot 10 \cdot 10^{-6} \mathrm{~K}$ & $5.75 \cdot 10^{-6} \mathrm{~K}^{-1} \cdot \theta \cdot L$ \\
\hline$L_{L I}$ & Eq. (7) & -1 & $\sqrt{0.23^{2}+\left(0.55 \mathrm{~K}^{-1} \cdot \Delta T\right)^{2}} \cdot 10^{-6} \cdot L$ \\
\hline$e_{\cos }$ & $0.005 \cdot 10^{-6} \cdot L$ & 1 & $0.005 \cdot 10^{-6} \cdot L \approx 0$ \\
\hline$e_{\text {DeadPath }}$ & max. $10 \mathrm{~mm}$ and $2 \mathrm{~K}$ & 1 & $\leq 10 \mathrm{~nm}$ \\
\hline & & \multicolumn{2}{|c|}{$u_{t o t}=45 \mathrm{~nm}+0.63 \cdot 10^{-6} \cdot L ;$ at $\theta \approx \Delta T \approx 0.1 \mathrm{~K}$} \\
\hline & & \multicolumn{2}{|c|}{$u_{\text {tot }} \leq 45 \mathrm{~nm}+6 \cdot 10^{-6} \mathrm{~K}^{-1} \cdot \theta \cdot L ;$ at $\theta>0.1 \mathrm{~K}$} \\
\hline
\end{tabular}

Table I: Uncertainty budget.

Where:

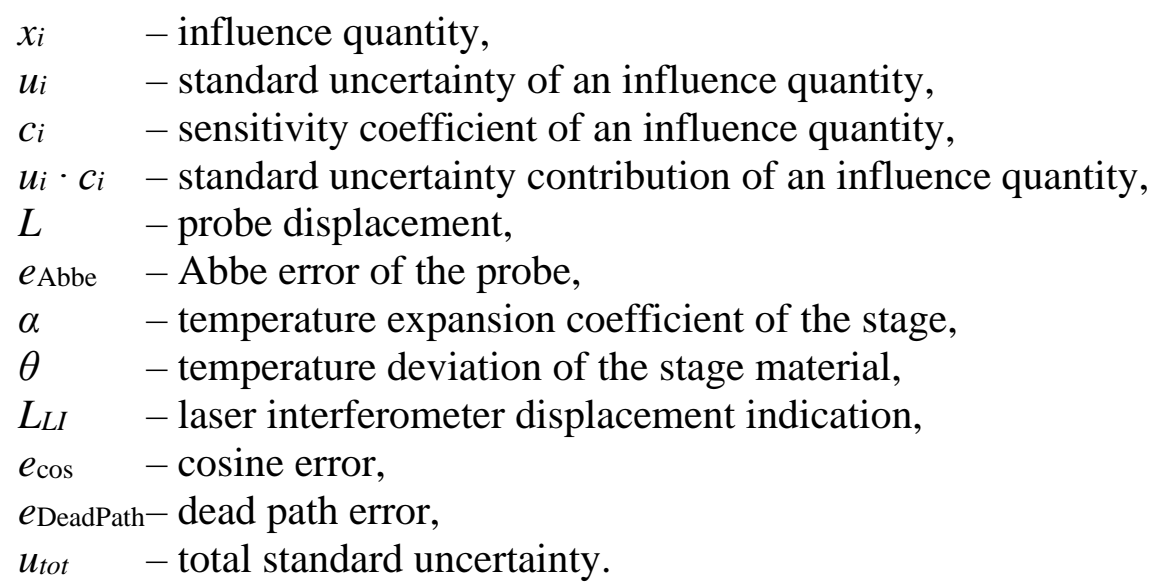

The uncertainty budget shows that even at relatively small temperature deviations (above $0.3 \mathrm{~K}$ ), the uncertainty due to the material expansion $u_{\text {mat }}=5.9 \cdot 10^{-6} \mathrm{~K}^{-1} \cdot \theta \cdot L$ becomes the ruling factor. For precise measurements, it is also very important that air conditions are well controlled to prevent air turbulence, which can influence the interferometry results.

Our measurement results $u_{\text {turb }}=0.04 \cdot 10^{-6} \cdot L$ showed that this component is negligible in comparison to the uncertainty $u_{\text {sensor }}=0.16 \cdot 10^{-6} \cdot L$, that was derived from the sensitivity of common sensors. Furthermore, the uncertainty component of the air in real industrial conditions generally exceeds the value $u_{a i r}=0.32 \cdot 10^{-6} \cdot \mathrm{L}$, that was calculated for our lab-controlled conditions, but still has minor influence in comparison to the material thermal component. 
Under such relatively well controlled lab-conditions it is also possible to include the air sensor into the laser-laser comparison test. Although the sensitivity of sensors is much smaller than the total uncertainty demonstrated by the experiment, its determination can still be good enough for industrial applications with material temperature deviations as predominant factor in the measurement accuracy.

\section{CONCLUSION}

Reliable measurement systems for complex geometrical measurements as well as for precise machine tool monitoring and verification are of great importance for modern sustainable manufacturing systems in sense of optimising production processes [20,21] and for controlling product quality. Since laser interferometers are often used for verifying machine tools and for calibrating important industrial measurement instruments, investigation of their precision and integration into manufacturing systems is of great importance.

Laser interferometers are very precise but also very delicate measurement instruments and shall be applied in well controlled measurement conditions. An operator should also be aware of all possible influences on the final precision of measurements. Systematic influences shall be minimised by precise measurement preparation and control.

Geometrical errors due to the installation can be significantly reduced or eliminated by proper alignment along the optical path. The environmental errors caused by laser's wavelength dependence on the air refractive index can be significantly reduced by stabilizing the air (temperature, air pressure, humidity). Instrumental error sources intrinsic to the laser system, such as electronic circuits, software algorithms, laser frequency stability, and polarization mixing, can be evaluated by separate calibrations of electronic-counting system and laser frequency. Another less precise way is to evaluate them together by the comparison with a reference laser.

The residual errors are comprised in the uncertainty of measurement. It is of significant importance that all non-compensated systematic errors, the residual compensated systematic errors and random errors are properly predicted and evaluated based on experiments, metrological knowledge and experiences.

\section{ACKNOWLEDGEMENT}

The authors acknowledge the financial support from the Slovenian Research Agency (research core funding No. P2-0190), as well as from Metrology Institute of the Republic of Slovenia (funding of national standard of length; contract No. C3212-10-000072).

\section{REFERENCES}

[1] Klobucar, R.; Acko, B. (2017). Automatic high resolution measurement set-up for calibrating precise line scales, Advances in Production Engineering \& Management, Vol. 12, No. 1, 88-96, doi:10.14743/apem2017.1.242

[2] Acko, B.; Weber, H.; Hutzschenreuter, D.; Smith, I. (2020). Communication and validation of metrological smart data in IoT-networks, Advances in Production Engineering \& Management, Vol. 15, No. 1, 107-117, doi:10.14743/apem2020.1.353

[3] Podzorny, T.; Budzyn, G.; Rzepka, J. (2013). Linearization methods of laser interferometers for pico/nano positioning stages, Optik, Vol. 124, No. 23, 6345-6348, doi:10.1016/j.ijleo.2013.05.054

[4] Haitjema, H. (2019). Calibration of displacement laser interferometer system for industrial metrology, Sensors, Vol. 19, No. 19, Paper 4100, 21 pages, doi:10.3390/s19194100

[5] Lipus, L. C.; Katic, M. (2020). Influence of intracavity power on frequency characteristics of iodine-stabilized He-Ne laser, Transactions of FAMENA, Vol. 44, No. 2, 23-30, doi:10.21278/ $\underline{\text { TOF. } 44203}$ 
[6] Lipus, L. C.; Budzyn, G.; Rzepka, J.; Acko, B. (2016). Calibration capability with laser frequency standard, Katalinic, B. (Ed.), DAAAM International Scientific Book 2016, DAAAM International, Vienna, 167-206, doi:10.2507/daaam.scibook.2016.18

[7] Lipus, L. C.; Matus, M.; Acko, B. (2013). Optimization of calibrating HeNe laser interferometers by sample-period simulation, International Journal of Simulation Modelling, Vol. 12, No. 3, 154163, doi:10.2507/IJSIMM12(3)2.231

[8] Klobucar, R.; McCarthy, M.; Acko, B. (2019). Metrological set-up for calibrating 2-dimensional grid plates with sub-micrometre precision, Measurement, Vol. 132, 60-67, doi:10.1016/ j.measurement.2018.09.035

[9] Birch, K. P.; Downs, M. J. (1994). Correction to the updated Edlén equation for the refractive index of air, Metrologia, Vol. 31, No. 4, 315-316, doi:10.1088/0026-1394/31/4/006

[10] Engineering Metrology Toolbox. Index of Refraction of Air, from https://emtoolbox.nist.gov/Wavelength/Abstract.asp, accessed on 05-02-2021

[11] Ciddor, P. E. (1996). Refractive index of air: new equations for the visible and near infrared, Applied Optics, Vol. 35, No. 9, 1566-1573, doi:10.1364/AO.35.001566

[12] Evaluation of Measurement Data. Guide to the Expression of Uncertainty in Measurement, from https://www.bipm.org/utils/common/documents/jcgm/JCGM_100_2008_E.pdf, accessed on 2402-2021

[13] Consortini, A.; Ronchi, L.; Stefanutti, L. (1970). Investigation of atmospheric turbulence by narrow laser beams, Applied Optics, Vol. 9, No. 11, 2543-2547, doi:10.1364/AO.9.002543

[14] Cai, Y. (2006). Propagation of various flat-topped beams in a turbulent atmosphere, Journal of Optics A: Pure and Applied Optics, Vol. 8, No. 6, 537-545, doi:10.1088/1464-4258/8/6/008

[15] Budzyn, G.; Rzepka, J. (2020). Study on noises influencing the accuracy of CNC machine straightness measurements methods based on beam position detection, Journal of Machine Engineering, Vol. 20, No. 3, 76-84, doi:10.36897/jme/127102

[16] Fan, W. G.; Zhang, S.; Wang, J. D.; Wang, X. H.; Wang, W. X. (2020). Temperature field of openstructured abrasive belt rail grinding using FEM, International Journal of Simulation Modelling, Vol. 19, No. 2, 346-356, doi:10.2507/IJSIMM19-2-CO10

[17] Safaric, J.; Dolsak, B.; Klobucar, R.; Acko, B. (2020). Analysis of thermal contribution to the measurement uncertainty in step gauge calibration, Precision Engineering, Vol. 66, 52-61, doi:10.1016/j.precisioneng.2020.06.012

[18] European Accreditation. EA-4/02 M: 2013 Evaluation of the Uncertainty of Measurement in Calibration, from https://european-accreditation.org/wp-content/uploads/2018/10/ea-4-02-mrev01-september-2013.pdf, accessed on 18-02-2021

[19] Stępień, K.; Talu, S. (2019). The study on influence of the method of handling of measuring head on measurement results obtained with the use of a portable profilometer, Technical Gazette, Vol. 26, No. 3, 592-595, doi:10.17559/TV-20160601124330

[20] Ojstersek, R.; Acko, B.; Buchmeister, B. (2020). Simulation study of a flexible manufacturing system regarding sustainability, International Journal of Simulation Modelling, Vol. 19, No. 1, 6576, doi:10.2507/IJSIMM19-1-502

[21] Malega, P.; Rudy, V.; Kanász, R.; Gazda, V. (2020). Decentralized optimization of the flexible production lines, Advances in Production Engineering \& Management, Vol. 15, No. 3, 267-276, doi:10.14743/apem2020.3.364 